\title{
El mobbing en la profesión periodística: análisis empírico del acoso psicológico en el trabajo y su influencia en el periodista y en la empresa informativa
}

\author{
Antonio LóPEZ HidALGO \\ Universidad de Sevilla \\ lopezhidalgo@us.es \\ María José UfARTE RUIZ \\ Universidad de Sevilla \\ mufarte@us.es
}

Recibido: 09/01/2013

Aceptado: 25/06/2013

\begin{abstract}
Resumen
Los casos de mobbing o acoso psicológico en el trabajo son frecuentes dentro de la profesión periodística, donde las amenazas, las presiones y las conductas hostigadoras se han convertido en compañeras de viaje de sus profesionales. El objetivo de este artículo científico es teorizar sobre esta práctica que se gesta, cada vez con mayor asiduidad, dentro de las redacciones informativas. La revisión crítica de la bibliografía existente, el análisis descriptivo de casos representativos, las entrevistas a los afectados y un estudio de caso aplicado a los profesionales de la prensa escrita de Almería, evidencian cómo el fenómeno afecta de forma insidiosa al bienestar físico y psicológico de los periodistas que lo padecen, suponiendo, al mismo tiempo, importantes costes económicos para las empresas informativas.
\end{abstract}

Palabras clave: periodistas, crisis, precariedad, acoso laboral, mobbing.

\section{The mobbing in the journalistic profession: Empirical analysis of the psychological harassment in the work and his influence in the journalist and in the informative company}

\begin{abstract}
The cases of mobbing or psychological harassment are frequent inside the journalistic profession at work, where the threats, the pressures and the harsh behavior have turned into partners for his professionals. The aim of this scientific article is to theorize on this practice that is happening, every time with major assiduity, inside the informative drafts. The critical review of the existing bibliography, the descriptive analysis of representative cases, the interviews to the affected ones and a study of case applied to the professionals of the written press of Almeria, demonstrate how the phenomenon concerns, in an alarming way, to the physical and psychological wellness of the journalists who suffer it, and at the same time, it assumes important economic costs to the informative companies.
\end{abstract}

Keywords: Journalists, crisis, precariousness, labor harassment, mobbing.

\section{Referencia normalizada}

LÓPEZ HIDALGO, Antonio y UFARTE RUIZ, María José (2013): "El mobbing en la profesión periodística: análisis empírico del acoso psicológico en el trabajo y su influencia en el periodista y en la empresa informativa”. Estudios sobre el Mensaje Periodístico. Vol. 19, Núm. 2 (julio-diciembre), págs.: 1017-1028. Madrid, Servicio de Publicaciones de la Universidad Complutense.

Sumario: 1. Introducción. 2. El mobbing en el ámbito periodístico. 3. Casos para el recuerdo. 4. Consecuencias del acoso laboral; 4.1. Repercusiones para el trabajador; 4.2. Repercusiones en la organización. 5. Propuesta de un modelo para evitar conductas de acoso. 6. Conclusiones. 7. Referencias. 


\section{Introducción}

Las denuncias por acoso laboral, hostigamiento psicológico o mobbing han aumentado en la profesión periodística de manera exponencial en los últimos años. Existe una tendencia a que determinados cargos de las redacciones informativas sometan a los periodistas a una presión e intimidación o les traten con violencia, lanzándoles incentivas e insultándoles, negándoles, en cualquier caso, todo tipo de respeto. En este contexto, Iñaki Piñuel (2006) ha advertido que los periodistas encabezan la lista de profesionales que padecen acoso moral en el trabajo, pues se encuentran en cuarta posición después de los funcionarios, los sanitarios y los educadores. El propósito de este estudio es analizar, desde una perspectiva cualitativa, esta denigrante situación cuyos efectos son patentes tanto a nivel personal como organizativo.

Con la finalidad de responder a estos objetivos, se ha recurrido a la revisión crítica de la bibliografía existente para el establecimiento de un marco teórico actualizado que permita visualizar las distintas perspectivas en torno a este fenómeno. El estudio incluye, igualmente, numerosas referencias a casos concretos y representativos que reflejan precisamente cómo los periodistas, imbuidos en todo el poder mediático que la sociedad les reconoce, son víctimas de violencia psicológica en su lugar de trabajo. Asimismo, incluimos datos propios derivados de entrevistas personales realizadas a tres periodistas víctimas de mobbing, Tomás Furest, Francisco Gil Chaparro y José Arroyo Gago, y cuyas experiencias reflejan la deshonrosa situación de acoso y trato vejatorio a la que estuvieron sometidos. Además, hemos llevado a cabo un pequeño estudio de caso que analiza la situación de los profesionales de la prensa escrita de Almería. Éstas, por tanto, han sido las principales fuentes informativas que se han utilizado en el desarrollo del presente trabajo y que han permitido que el mismo cuente con una metodología pertinente.

Tras la introducción, el trabajo se divide en cinco apartados. En el primero de ellos, revisamos este concepto y su vinculación al ámbito informativo. En el capítulo segundo, nos adentramos en diferentes casos que evidencian cómo los periodistas acosados laboralmente son numerosos. Tras el análisis de estos casos concretos, en el apartado tercero, reflejamos las consecuencias que conlleva este fenómeno tanto para el trabajador como para la organización y, finalmente, en el capítulo cuarto proponemos diferentes métodos para sortear la aparición de este tipo de conductas en las redacciones informativas. En el apartado de conclusiones incluimos los principales resultados de la investigación e identificamos los nuevos parámetros que deben ser considerados por las empresas informativas.

\section{El mobbing en el ámbito periodístico}

Por mobbing se entiende actos o comportamientos discriminatorios o vejatorios protagonizados en el tiempo, y llevados a cabo, en el ámbito del trabajo dependiente, público o privado, por parte del empresario o sus subordinados o bien por parte de otros compañeros, y que se caracteriza como una verdadera y propia forma de persecución psicológica o de violencia moral (Correa Carrasco, 2006: 79). En España, quien más ha investigado sobre este tema ha sido el psicólogo y profesor Iñaki Piñuel que, con el objetivo de evaluar el mobbing en el entorno laboral de las organizaciones y sus con- 
secuencias, elaboró en el año 2001 el barómetro Cisneros, primera herramienta que se utilizó para medir la incidencia del fenómeno del acoso psicológico en nuestro país ${ }^{1}$.No obstante, se trata de un fenómeno surgido en la década de los ochenta por el psicólogo alemán Heinz Leyman y cuyo origen o naturaleza es heterogénea. Pese a que influyen diferentes agentes, el substrato que favorece la aparición de ese tipo de conductas se encuentra ligado a una organización deficiente y con pobre método de trabajo, con la existencia de múltiples jerarquías y cargas excesivas debido a la escasez de plantilla (Leyman, 1996: 65). Marie France Hirigoyen, pionera en el estudio del acoso laboral en Francia, incide en aspectos afines:

Estas conductas se ven favorecidas en organizaciones con un método de trabajo y producción con organización extremadamente pobre, con ausencia de interés y apoyo por parte de los superiores, con ausencia de relación entre éstos, con ausencia de posibilidades de colaboración o mejoras relativas en cuestiones personales, con la existencia de múltiples jerarquías, con cargas excesivas de trabajo debido a la escasez de plantilla o mala distribución de la misma, con una deficiente organización diaria del trabajo, con la existencia de líderes espontáneos no oficiales, con un trabajo con bajo contenido, monótono y con escasa autonomía, con conflictos de rol, y con flujos pobres de información (Hirigoyen, 2006: 92).

Si aplicamos estos factores causales al ámbito periodístico observamos cómo las condiciones de trabajo existentes en determinadas redacciones informativas, y recientemente Internet, favorecen la aparición de ciertas conductas de hostigamiento psicológico. Además, la crisis que afecta al sector desde al menos el año 2008 no ha hecho sino empeorar este escenario. En primer lugar, la organización del trabajo en el seno de la profesión periodística se encuentra desnutrida y raquítica. José Alonso Seco (2003: 89) describe muy bien este escenario cuando afirma que en las redacciones informativas además de encontrar a un gran número de becarios hay profesionales que trabajan sin tener contrato. De igual manera, en los medios convencionales, asfixiados y saturados por el descenso de ingresos publicitarios, las reorganizaciones empresariales y los ajustes de plantilla, la mayoría de las nuevas contrataciones se traducen en reemplazos de periodistas veteranos por jóvenes con contratos precarios. En este sentido, a las tradicionales categorías profesionales de director, subdirector, redactor jefe, jefe de sección y redactor, hay que sumar otras fórmulas de empleo alternativas, pero menos estables como auxiliar de redacción, ayudante de redacción, colaborador, becario, y estudiantes en prácticas. Esta diversidad de cargos repercute, igualmente, en el hecho de que el profesional de la información derive, modifique o varíe sus quehaceres diarios.

1 El barómetro Cisneros es un cuestionario elaborado por el profesor Iñaki Piñuel y Zabala para sondear de manera periódica el estado y los índices de violencia en el entorno laboral. Está construido de modo que las personas que lo contestan en el orden propuesto no saben, de forma explícita, hasta la pregunta número 47 que los comportamientos sobre los que se les ha consultado anteriormente en la parte del barómetro tipifican un fenómeno descrito como mobbing o acoso psicológico en el trabajo. El barómetro Cisneros se aplicó por primera vez a trabajadores en activo de todos los sectores en la Comunidad de Madrid en el entorno de Alcalá de Henares y Guadalajara. Fue la primera investigación monográfica con una muestra amplia realizada en España con casi 1.000 encuestas válidas. 
Las altas cargas de trabajo también son muy comunes dentro del ámbito periodístico y es un hecho que esta situación está afectando al bienestar del profesional de la información. En la profesión, los tiempos son implacables y estar a la vanguardia de la información y trabajar rápidamente por el temor de perder una noticia de interés o de lanzar una información que no esté suficientemente verificada, provocan situaciones límite. María José Canel, Roberto Rodríguez Andrés y José Javier Sánchez (2000: 24) han señalado al respecto que las jornadas de trabajo se estiran hasta bien entrada la noche y las prisas y el estrés se han convertido en compañeros habituales del periodista. Son muchos los autores que se han pronunciado en esta línea advirtiendo que los periodistas tienen demasiadas cosas por hacer y todas de alta prioridad. Además, hay que tener en cuenta que su situación laboral no es la idónea debido a que soportan sacrificios salariales y laborales para salvaguardar su empleo (APM 2011: 73).

La satisfacción laboral, según Fidalgo Vega (2002), también es determinante a la hora de que se geste el mobbing. Desde el punto de vista periodístico, existe un alto nivel de insatisfacción debido principalmente a la estabilidad del empleo (61\%), a la flexibilidad de los horarios (36,3\%), posibilidades de promoción (35,0\%), grado de autonomía (24,8\%), y cobertura de otras áreas informativas (16,2\%) (APM, 2009: 33 ). Se trata de un sentimiento que aumenta con el paso de los años pues, si en 2008 la tasa de insatisfacción era del 39,5 por ciento, en 2009 se sitúa en el 40,8 por ciento. Asimismo, el grado de satisfacción económica de los periodistas se sitúa en 5,4 puntos en una escala del 1 al 10 (APM, 2009: 34-37). La importancia del contexto laboral viene dada, principalmente, porque los procesos son deudores, en gran medida, de deficiencias constatables en el plano organizativo.

Por otro lado, y en lo que se refiere a los sectores, Hirigoyen (2006: 110) sostiene que el acoso psicológico es más brutal en el sector privado ya que dura menos y suele terminar con la marcha de la víctima de la empresa. Por el contrario, señala que en el sector público el acoso moral puede durar varios años debido a que, en principio, las personas están protegidas y no pueden ser despedidas a menos que cometan una falta grave. En similares términos se pronuncia Marisa Bosqued:

En la empresa privada el hostigamiento psicológico suele ser más brutal y de más corta duración en el tiempo: el empleado se va, le echan. Como el objetivo perseguido se consigue en un periodo temporal más breve, deja de ser necesario un largo e insidioso proceso de acoso. En cambio, en la Administración, el mobbing es más prolongado en el tiempo, adoptando a veces formas kafkianas. (Bosqued Lorente, 2005: 42)

Como queda demostrado, la profesión periodística se ha degradado seriamente y sus profesionales poseen unas pésimas condiciones laborales, como se evidencia en su modalidad de contratación laboral, en sus salarios o bien en los prolongados horarios. La situación conduce directamente a la aparición de ciertas conductas de acoso $\mathrm{y}$, por consiguiente, a que sean numerosos los profesionales que afirman padecerlas.

\section{Casos para el recuerdo}

Aunque la sociedad ensalza normalmente al periodista como un adalid de la libertad de expresión, éste no siente un impulso a la hora de reivindicar sus propios derechos 
como trabajador y denunciar el acoso y las presiones a las que está sujeto. (López Hidalgo, Fernández Barrero, Romero Portillo, 2010). La denuncia por las amenazas que sufren ha sido, hasta hace poco años, un tema prohibido y desterrado. De hecho, no será hasta 2002 cuando se consiga una de las primeras sentencias favorables y se cree jurisprudencia en el marco del acoso laboral. La nueva legislación llega de la mano de Gerardo Mediavilla, un periodista que durante varios años es víctima de acoso laboral mientras trabaja en el Departamento de Prensa del Ayuntamiento de Madrid. Su caso sirve para que muchos otros periodistas denuncien este fenómeno que tiene una clara relevancia social y afecta de forma insidiosa al bienestar físico y psicológico de muchos profesionales de la información. Mediavilla ha afirmado: "Ninguna sentencia va a restituirme el sufrimiento y la pesadilla que viví durante esos tres años, pero me alegro de que los jueces se pronuncien y de que otras personas se animen a denunciar a sus empresas" (en Lucio, 2003).

El hecho de que salga a la luz ha conseguido que las denuncias por acoso laboral hayan ido creciendo en el seno de la profesión. De esta manera, otro caso llamativo es el del periodista Tomás Furest, que fue acosado laboralmente en 2001 por el entonces director de El Correo de Andalucía, Fernando Orgambides. Su historia es un caso claro de mobbing sentenciado así por el Juzgado de lo Social n ${ }^{\circ} 9$ de Andalucía. En la sentencia 233/03 queda reflejada la situación de acoso y trato vejatorio a la que estuvo sometido durante casi tres años:

Todo comienza cuando nombran como director en El Correo de Andalucía a Fernando Orgambides. Yo, por aquel entonces, era redactor jefe de Deportes y, hasta la fecha y debido a mi larga trayectoria laboral en el mundo periodístico, gozaba de respeto profesional y me sentía apreciado por mis compañeros (Ufarte, M.J.: entrevista a Tomás Furest, ex redactor jefe de la sección de Deportes de El Correo de Andalucía, 24 de septiembre de 2009).

La historia de Tomás Furest no se produce de manera aislada en El Correo de Andalucía. El periodista Francisco Gil Chaparro también sufrió conductas de acoso por parte de Fernando Orgambides:

Desde su llegada al periódico, emprendió una campaña de acoso laboral que afectó, sin excepción, a toda la cúpula de la redacción, desde el director que le precedió, a los subdirectores, redactores jefes y jefes de sección, entre los que se encontraban Antonio Ramos Espejo, Antonio Avendaño, Rafael Guerrero o Sebastián García (Ufarte, M.J.: entrevista a Francisco Gil Chaparro, ex redactor jefe de la sección de Cultura de El Correo de Andalucía, 14 de diciembre de 2012).

En 2003, también salta a la luz el caso de José Arroyo Gago, víctima de mobbing mientras trabaja como responsable de prensa en la Delegación Territorial de la Junta de Castilla y León en Salamanca:

Todo comenzó cuando entró un nuevo secretario territorial. Hacía y deshacía a su antojo, ejercía de censor y me prohibía hablar con los colegas de los medios que no eran de su cuerda, más otras actitudes nada democráticas. El diálogo era imposible porque se negaba hasta que le advertí que, si seguía tratándome de esa manera, le denunciaría. Y ahí comenzó el acoso. Cavé mi tumba. Poco a poco, como en un martirio chino, me convirtió en una silla más de mi despacho. Siempre en la sombra (Ufarte, M.J.: entrevista a José Arroyo 
Gago, ex responsable de prensa en la Delegación Territorial de la Junta de Castilla y León en Salamanca, 9 de octubre de 2010).

Estos casos no son únicos dentro de la profesión periodística. El portal digital mallorcadiario.com publica en 2007 una noticia donde una redactora del periódico impreso gratuito de Mallorca denuncia ante la Policía Nacional al director de la publicación por acoso laboral y comentarios sexuales vejatorios (mallorcadiario, 2007). De la misma manera, el periodista y presentador Carlos García Hirschfeld ha denunciado a Antena 3 Televisión por acoso moral al considerar que la cadena del grupo Planeta utiliza de modo sesgado el derecho de los trabajadores a la excedencia laboral: "Esta es la táctica de la empresa para tratar de aburrir a los trabajadores veteranos para que nos marchemos y ahorrarse la indemnización" (elconfidencialdigital, 2007). El periodista de espacios musicales Ramón Trecet también ha hecho público el acoso laboral sufrido en Radio Nacional por parte de su directora (Piña, 2008).

En numerosas ocasiones, el argumento central de esta situación acaba siendo el pésimo estado en el que se encuentra el mercado laboral periodístico que reúne características desfavorecedoras. Para Ángel Fernández (2009) cuando hay un exceso de oferta de trabajadores, los directivos pueden caer en la tentación de pensar que su sustitución es fácil e introducir conductas de cierto acoso si a alguna persona no le interesa. Los trabajadores, por su parte, se sienten más indefensos y se aferran a sus puestos casi a cualquier precio. No hay que olvidar que España es el país con mayor contratación temporal de la Unión Europea y uno de los que presenta mayores tasas de paro. Además, en muchas empresas periodísticas no existe convenio colectivo y sus ordenanzas laborales son obsoletas; la jornada laboral no se respeta de manera sistemática y los colaboradores y freelances se ven a menudo forzados a trabajar sin contrato y sin cobertura legal alguna. A estas circunstancias, según López Hidalgo, Fernández Barrero y Romero Portillo (2010) hay que sumar otras necesidades: la regulación de las prácticas de licenciados y no licenciados y de la actividad de los horarios universitarios; la regulación de la figura del periodista al servicio de las Administraciones Públicas; la regulación y cumplimiento de los derechos de autor de todos los periodistas; la defensa de la existencia de medios públicos de comunicación, de su objetividad y pluralismo informativos y de un sistema transparente y profesional de acceso a los puestos de trabajo; o la existencia de Consejos de Redacción en los medios de comunicación, regulados por Estatutos de Redacción, que tengan vigentes un Código Deontológico.

En lo que respecta a los actos de hostigamiento, éstos se pueden presentar de forma activa, reflejándose mediante gritos, insultos, represiones, humillaciones en público y en privado. Así lo ha destacado Tomás Furest:

En varias ocasiones, Orgambides llegó a la redacción del periódico gritando y acusándome de querer hundir el periódico, de la pérdidas de ventas, y de ser un abuelo y estar acabado. Todo ello mediante insultos y en presencia de todo el personal de la redacción. Su acoso iba en aumento. Me gritaba delante de mi equipo y me decía que hasta un becario lo haría mejor. (Ufarte, M.J.: entrevista a Tomás Furest, ex redactor jefe de la sección de Deportes de El Correo de Andalucía, 24 de septiembre de 2009). 
En la misma línea se pronuncia Francisco Gil Chaparro:

Menospreciaba mi trabajo delante de los compañeros y se dirigía a mí mediante gritos y desplantes, minusvalorando mi capacidad profesional. Además, me hacía esperar hasta las primeras horas de la madrugada y revisaba una a una las páginas de Cultura, que era la sección que yo dirigía. (Ufarte, M.J.: entrevista a Francisco Gil Chaparro, ex redactor jefe de la sección de Cultura de El Correo de Andalucía, 14 de diciembre de 2012).

También pueden manifestarse mediante el aislamiento del trabajador:

Me cortó el teléfono, cambió el rótulo de mi despacho donde se leía prensa por otro, donde figuraba Protección Civil. Convirtió mi oficina en un basurero, me eliminó de la lista telefónica interna y comenzó a encargarse él de mis labores. Salvo contadísimas excepciones, la mayoría de mis compañeros me rehuían; dejaron de salir a tomar café conmigo y hasta los hubo que dejaron de hablarme. El colmo fue cuando algunos se prestaron a seguir haciéndome la vida imposible y a irle a contar al jefe las cosas que yo hacía o dejaba de hacer (Ufarte, M.J.: entrevista a José Arroyo Gago, ex responsable de prensa en la Delegación Territorial de la Junta de Castilla y León en Salamanca, 9 de octubre de 2010).

Los periodistas almerienses de la prensa escrita también se han pronunciado en esta línea advirtiendo que sufren ciertas situaciones de hostigamiento psicológico. Así lo revela un estudio de caso de elaboración propia ${ }^{2}$ donde se pone de manifiesto que un $41 \%$ es, a veces, víctima de gritos o insultos delante de sus compañeros por cuestiones laborales. Igualmente, un $21 \%$ sufre a veces acoso psicológico en el lugar de trabajo por parte de algún superior. Respecto a la posibilidad de si alguna vez habían menospreciado sus superiores su trabajo por razones ideológicas, religiosas, políticas o de sexo, un $44 \%$ de la muestra respondió que nunca frente a un $27 \%$ que señaló que a veces.

De todos estos datos se desprende que en el seno de la profesión periodística el acoso psicológico o moral se ha disparado y, como apunta la Asociación Nacional de Entidades Preventivas Acreditas (ANEPA), afecta ya a un diez por ciento de los trabajadores españoles (Calero, 2003: 6). Como consecuencia, la sociedad ha empezado a cobrar conciencia de la realidad de este problema, por lo que en los últimos tiempos han aparecido una serie de profesionales tales como especialistas en medicina del trabajo, juristas, sindicalistas, psiquiatras, psicólogos y psicosociólogos intentando apuntar soluciones al respecto. Igualmente, se han creado asociaciones de ayuda a las víctimas.

\section{Consecuencias del acoso laboral}

El mobbing o acoso moral en el trabajo es especialmente dañino para la salud del trabajador y el bienestar de la organización. No obstante, no tiene las mismas conse-

2 Estudio de caso sobre la situación laboral realizado en febrero de 2011. MUESTRA: 82 periodistas de la prensa escrita de Almería de los que un 46\% son mujeres y un 36\% hombres. Los periodistas se distribuyen en tres medios: La Voz de Almería, que aglutina al 37\% de los periodistas encuestados; Diario de Almería con el 26\% e Ideal, con el 19\% restante. Los periodistas se sitúan en una franja de edad comprendida entre los 30 y los 40 años. 
cuencias ni provoca las mismas reacciones en todas las personas debido, fundamentalmente, a que las diferencias entre las habilidades, capacidades y recursos para afrontarlas pueden ser muy distintas.

\subsection{Repercusiones para el trabajador}

Los daños que el mobbing produce en las víctimas son numerosos y considerables. Sin embargo, la dificultad de cuantificar tales daños estriba en el hecho de que, en cierta medida, estamos ante un aspecto impregnado de una gran dosis de subjetividad. A nivel general, podemos hablar de una sintomatología que se manifiesta a través del estrés, ansiedad, insomnio, irritabilidad, híper vigilancia, fatiga, cambios de personalidad, depresión y trastornos psicosomáticos. No obstante, los síntomas afectan a tres niveles: psíquico, físico y social. Aunque están relacionados entre sí, es el nivel psíquico el que mayor protagonismo cobra debido a que los síntomas más comunes son el estrés y la ansiedad. Ahora bien, dependiendo de la fase en la que se encuentre cada víctima y de la duración e intensidad de la misma, se pueden desarrollar unos efectos u otros. El periodista Tomás Furest, por ejemplo, reconoce:

Padecía un trastorno adaptativo mixto ansioso depresivo de carácter agudo, elevado tono emocional, ansiedad, visión negativa de mí mismo, de mi presente y de mi futuro, sentido del humor perdido, depresión y sensación inexplicable de culpabilidad. Además perdí la ilusión por el mundo del trabajo y la sociedad en general (Ufarte, M.J.: entrevista a Tomás Furest, ex redactor jefe de la sección de Deportes de El Correo de Andalucía, 24 de septiembre de 2009).

\section{Por su parte, Francisco Gil Chaparro manifiesta:}

Los médicos especialistas me dieron una baja laboral por depresión. Padecía ansiedad y alteración de la afectividad (Ufarte, M.J.: entrevista a Francisco Gil Chaparro, ex redactor jefe de la sección de Cultura de El Correo de Andalucía, 14 de diciembre de 2012).

A esta sintomatología, a nivel general, habría que añadir la pérdida de ilusión por el mundo del trabajo y la sociedad. En opinión de Hirigoyen (2006: 148) el mobbing consigue, en definitiva, desgastar, usar y minar a las personas, que pierden las ilusiones y la esperanza. A nivel físico, las consecuencias también son devastadoras y se traducen en llanto, vómitos, mareos, desmayos, dolores de cabeza, dificultades en la respiración y fatiga ante el más mínimo esfuerzo. Por otro lado, el entorno social del acosado también padece las consecuencias de tener una persona cercana amargada, desmotivada y sin expectativas ni ganas de trabajar (Fidalgo, 2002). Por su parte, la familia de la víctima también se ve afectada debido a que las alteraciones psicológicas y psiquiátricas que padece el acosado dificultan las relaciones en el hogar y facilita la aparición de conflictos. En opinión de Piñuel (2005: 140) la víctima sufre una desestabilización grave y alteraciones emocionales y de la personalidad que afectan a su esfera de relaciones sociales y familiares, generando problemas de relación social y de pareja que no existían anteriormente:

La situación era insostenible y empezaba a afectar a mi familia que era consciente de la persecución a la que estaba sometido. Yo estaba muy irritable y saltaba por cualquier cosa. Pagaba mi malestar con ellos sin tener el más mínimo motivo (Ufarte, M.J.: entrevista 
a Tomás Furest, ex redactor jefe de la sección de Deportes de El Correo de Andalucía, 24 de septiembre de 2009)".

Al hilo de esta situación, Marisa Bosqued (2005: 175) informa que la pareja de la víctima tiene un peso muy importante ya que puede realizar un espléndido papel terapéutico sirviéndole de apoyo y de guía en el proceso de superación. Por otro lado, es importante destacar que la situación de acoso a las que están sometidos los periodistas no solo afecta a la dignidad del colectivo sino que también repercute en la manera en que éstos desarrollan su trabajo y, por consiguiente, en la forma en que la información llega a los ciudadanos.

\subsection{Repercusiones en la organización}

Además de causar determinados sufrimientos a las personas que lo padecen, el mobbing también tiene una serie de consecuencias para la organización. Por lo general, los efectos que produce en las empresas se centran en disminuir la calidad, la producción y el rendimiento. Como señala Tomás Furest:

Hubo un momento en el que hasta hacer un breve se me hacía una montaña, como si nunca hubiera trabajado en un periódico (Ufarte, M.J.: entrevista a Tomás Furest, ex redactor jefe de la sección de Deportes de El Correo de Andalucía, 24 de septiembre de 2009).

Igualmente, este hecho se traduce en unos textos periodísticos menos elaborados, superficiales y epidérmicos que dejan al margen la investigación y el contraste. En esta línea, los expertos no han titubeado a la hora de señalar que la difícil situación por la que atraviesan estos profesionales desemboca en un periodismo pobre, trivial y sensacionalista donde se olvida la investigación a favor de las informaciones procedentes de las agencias de noticias, de las oficinas de prensa, de las instituciones públicas o bien de las literales declaraciones de los diferentes personajes. A ello hay que sumar los efectos de la precariedad laboral que han dado lugar a una parcialidad periodística que ha sustituido la calidad por la cantidad. La enorme cantidad de datos, declaraciones y opiniones de las que se componen diariamente los medios de comunicación producen en el público correspondiente la sensación de pluralidad.

El enrarecimiento en el clima laboral también es consecuencia directa del mobbing. La periodista Elena de Regoyos (2006) recuerda la situación que se vivió en $L a$ Razón cuando salieron a la luz las famosas listas negras y las bajas producidas en el medio como consecuencia de este fenómeno. Según Regollos, la situación en el periódico era bastante mala ya que el informe creó una sensación de estado policial y de desconfianza que provocó que nadie se fiara de nadie. Era como una caza de brujas donde predominaban los despidos y las bajas.

En cualquier caso, el desenlace habitual de la situación de mobbing suele significar la salida de la víctima de la organización de manera voluntaria o forzosa. Así lo afirma Francisco Gil Chaparro:

Tras la presentación de la demanda, la empresa reaccionó despidiéndome de forma fulminante. Con posterioridad, y a través de la figura del gerente, trataron de negociar una salida al conflicto laboral antes de que se llegara a juicio, que había sido señalado para los 
primeros días del mes de enero de 2003. La oferta de la empresa consistió en ofrecerme una cantidad de dinero muy pequeña y la posibilidad de que me acogiera al paro, propuesta que rechacé. La aceptación, finalmente, por parte de la empresa y mía fue negociar un despido improcedente, que puso fin al conflicto justo una semana antes de que se celebrara el juicio por acoso laboral (Ufarte, M.J.: entrevista a Francisco Gil Chaparro, ex redactor jefe de la sección de Cultura de El Correo de Andalucía, 14 de diciembre de 2012).

La empresa, por su parte, daña su imagen pública sobre todo cuando estos supuestos trascienden a los medios de comunicación. Los costes que conlleva en la sociedad también son importantes. En España ya se ha empezado a tomar conciencia y se han estudiado y analizado los gastos de las mutuas de accidentes de trabajo y enfermedades profesionales de la Seguridad Social. En este sentido, destaca el análisis llevado a cabo por Ibermutuamur que investigó sobre 6.500 bajas, de las cuales un $1,71 \%$ pertenecían a este tipo de acoso. Estos datos, traducidos en cifras más comunes, suponen 1.850.000 días perdidos y más de 52.000 millones de euros por año (Pastrana Jiménez, 2002: 176).

\section{Propuesta de un modelo para evitar conductas de acoso}

La erradicación del mobbing debe contemplarse desde una perspectiva triple que requiere del compromiso de diferentes agentes. En primer lugar, los responsables de las empresas informativas deben mejorar el entorno laboral y la organización del trabajo. Las asociaciones de profesionales, por su parte, deben desarrollar un marco legislativo más amplio y concreto para éste y otros tipos de prácticas similares. Por supuesto, los periodistas deben denunciar cualquier tipo de maltrato dentro del ámbito laboral. No obstante, el primer paso para su eliminación pasa por evitar cualquier tipo de conducta hostigadora dentro de las redacciones informativas. Para ello, planteamos el siguiente decálogo de propuestas:

1. Incremento de la productividad del trabajador, por la vía de la lucha contra la organización deficiente.

2. Crear una estructura organizativa que ayude al logro de los objetivos de la empresa con un mínimo de consecuencias o costos no deseados.

3. Favorecer el interés de los trabajadores por la actividad profesional.

4. Apoyo y reconocimiento del trabajo bien realizado por parte de los superiores.

5. Aumentar la relación con los superiores y frenar el liderazgo.

6. Apostar por una comunicación más fluida y menos jerárquica.

7. Diversificar las cargas de trabajo mediante procedimientos bien organizados y no rutinarios.

8. Potenciar el sentido crítico de la profesión periodística.

9. Aumentar la autonomía e independencia de sus profesionales.

10. Ejercer la profesión de una manera digna y con unos sueldos asequibles.

\section{Conclusiones}

El número de periodistas acosados laboralmente es, cada vez, más significativo tal y como ha quedado demostrado en los diferentes casos descriptivos aportados y en las 
entrevistas realizadas a tres de los afectados, Tomás Furest, Francisco Gil Chaparro y José Arroyo Gago. Además, los profesionales de la información son protagonistas de acusaciones injustificadas o de falsos incumplimiento y errores que no tienen consistencia ni entidad real. Las causas de este fenómeno se localizan en una mala organización de las empresas informativas, en las excesivas cargas de trabajo y en los numerosos rangos profesionales de las redacciones.

En cuanto a las consecuencias son patentes tanto a nivel personal como profesional. A nivel personal, repercute en una mala salud del trabajador y, a nivel organizativo, provoca enrarecimiento del clima laboral y afecta a la calidad de los textos periodísticos que se presentan ante la sociedad receptora. En definitiva, una empresa que cuente con una idónea organización de los puestos de trabajo y con unos superiores que controlan lo que ocurre y se preocupan del ambiente laboral el mobbing no va a ser proclive, puesto que las circunstancias impiden el desarrollo del mismo.

\section{Referencias}

ALONSO SECO, José (2003): "El mejor oficio, si se deja a tiempo" en LARRAÑAGA ZUBIZARRETA, José y DÍAZ NOCI, Javier (coords.): El periodista ante el mercado laboral. Bilbao, Universidad del País Vasco.

ASOCIACIÓN DE LA PRENSA DE MADRID (2010): Informe anual de la profesión periodística 2009. Madrid, APM.

ASOCIACIÓN DE LA PRENSA DE MADRID (2012): Informe anual de la profesión periodística 2011. Madrid, APM.

BOSQUED LORENTE, Marisa (2005): Mobbing. Cómo prevenirlo y superar el acoso psicológico. Barcelona, Paidós.

CALERO JAÉN, Carlos (2003): El mobbing o acoso psicológico en el trabajo. Comisión ejecutiva nacional de la Unión General de Trabajadores de Valencia. Valencia, Ediciones UGT-PV.

CANEL, María José; RODRÍGUEZ, Roberto; SÁNCHEZ, José Javier (2000): Periodistas al descubierto. Retrato de los profesionales de la información, Madrid, Centro de Investigaciones Sociológicas.

CORREA CARRASCO, Manuel. (2006): "El concepto de acoso moral en el trabajo". En Acoso Moral en el Trabajo. Concepto, prevención, tutela procesa y reparación de daños. Navarra, Colección Monografías Aranzadi.

DE REGOYOS, Elena (2006): “¿Bajas por mobbing en La Razón?”. En www.periodistadigital.com. 15 de febrero de 2006.

Confidencialdigital.com (2007): "El periodista Carlos García Hirschfeld denuncia a Antena 3 TV por 'mobbing' tras ser trasladado a Canarias". En www.confidencialdigital.com, 2 de noviembre.

FIDALGO VEGA, Manuel (2002): "Acoso psicológico en el trabajo: mobbing”. Ponencia en el Acto de Graduación del curso de nivel intermedio en Prevención de Riesgos Laborales, Barcelona. 
mallorcadiario.com (2007): "Una periodista denuncia a su director por acoso". En mallorcadiario.com, 22 de febrero.

HIRIGOYEN, Marie France (2006): El acoso moral en el trabajo, distinguir lo verdadero de lo falso. Paidós, Barcelona.

LEYMAN, Heinz (1996): La persécutionautravail. Ed. Du Seuil, Paris.

LÓPEZ HIDALGO, Antonio; FERNÁNDEZ BARRERO, Ángeles.; ROMERO PORTILLO, José (2010): "Facebook, en defensa de la profesión periodística", Comunicación presentada en el XII Congreso de la SEP. Periodística y web 2.0: hacia la construcción de un nuevo modelo. Valencia, 21 y 22 de mayo de 2010.

LUCIO, Cristina (2003): "Gerardo Mediavilla: El mobbing es clandestino". En El Mundo, 19 de junio de 2003.

MÉNDEZ Juan (2006): "Evolución y cambios en la información sociolaboral: de la especialización a la generalización" en Jornadas sobre Relaciones Laborales y Medios de Comunicación. Sevilla, 12/12/06.

PASTRANA JIMÉNEZ, José Ignacio (2002): "Cuánto cuesta el mobbing en España". En Revista de Relaciones Laborales, núm. 7.

PIÑA, Raúl (2008): "Ramón Trecet: me están haciendo mobbing en RNE”. En www.elmundo.es, 24 de octubre de 2008.

PIÑUEL y ZABALA, Iñaki (2006): "Funcionarios, sanitarios y periodistas son las principales víctimas de mobbing". En www.lagacetadecanarias.net, 1 de julio. PINUEL y ZABALA, Iñaki (2005): Mobbing, manual de autoayuda. Afronte el acoso psicológico en el trabajo. Barcelona, Debolsillo.

UFARTE RUIZ, María José (2009): Entrevista realizada a Tomás Furest. (24/09/2009).

UFARTE RUIZ, María José (2010): Entrevista realizada a José Arroyo Gago. $(09 / 10 / 2010)$.

UFARTE RUIZ, María José (2012): Entrevista realizada a Francisco Gil Chaparro. $(14 / 12 / 2012)$. 